\title{
Cardiovascular risk prediction: the old has given way to the new but at what risk-benefit ratio?
}

This article was published in the following Dove Press journal:

Research Reports in Clinical Cardiology

14 October 2014

Number of times this article has been viewed

\section{Joseph Yeboah}

Heart and Vascular Center of Excellence, Wake Forest University School of Medicine, Winston-Salem, NC, USA
Correspondence: Joseph Yeboah Heart and Vascular Center of Excellence, Wake Forest University School of Medicine Medical Center Blvd, Winston Salem, NC 27I57, USA

Tel +l 3367167015

Fax+I 3367169188

Email jyeboah@wakehealth.edu

\begin{abstract}
The ultimate goal of cardiovascular risk prediction is to identify individuals in the population to whom the application or administration of current proven lifestyle modifications and medicinal therapies will result in reduction in cardiovascular disease events and minimal adverse effects (net benefit to society). The use of cardiovascular risk prediction tools dates back to 1976 when the Framingham coronary heart disease risk score was published. Since then a lot of novel risk markers have been identified and other cardiovascular risk prediction tools have been developed to either improve or replace the Framingham Risk Score (FRS). In 2013, the new atherosclerotic cardiovascular disease risk estimator was published by the American College of Cardiology and the American Heart Association to replace the FRS for cardiovascular risk prediction. It is too soon to know the performance of the new atherosclerotic cardiovascular disease risk estimator. The risk-benefit ratio for preventive therapy (lifestyle modifications, statin +/- aspirin) based on cardiovascular disease risk assessed using the FRS is unknown but it was assumed to be a net benefit. Should we also assume the risk-benefit ratio for the new atherosclerotic cardiovascular disease risk estimator is also a net benefit?
\end{abstract}

Keywords: risk prediction, prevention, cardiovascular disease

\section{Introduction}

Cardiovascular disease (CVD) has been and is still the leading cause of death in the developed world despite the significant progress made in the identification of risk factors, reduction of the prevalence of risk factors, and treatment. ${ }^{1}$ Further reduction in the incidence and prevalence of CVD will require better identification of at risk individuals for proven therapies. The identification of at risk individuals in our populations for appropriate therapy to reduce this risk falls under two broad umbrellas: primary and secondary CVD prevention. ${ }^{2}$

\section{Discussion}

Secondary prevention involves reducing subsequent CVD events in individuals who already have the disease. Currently all individuals with clinical CVD are considered high risk for subsequent CVD events and treated as such with available proven therapies. In such individuals, there is no recommended further risk stratification. However, for free living asymptomatic individuals without prior CVD, there exists a wide continuum of risk for CVD events. Hence proven therapies have to be recommended for this very large subgroup of the population based on risk-benefit ratios to prevent primary CVD events (primary prevention). Primary cardiovascular risk 
prediction involves the utilization of tools to identify a subgroup of asymptomatic individuals without prior CVD to whom the application of current proven lifestyle and medicinal therapies would yield a reduction in CVD events and a net benefit to society.

The concept of cardiovascular risk prediction dates back to 1976 when the Framingham coronary heart disease (CHD) risk score was published. ${ }^{3}$ The Framingham Heart study investigators used traditional risk factors to derive a risk equation in a Caucasian population to predict an individual's 10 year risk of having a CHD event. The widespread utilization of this tool however did not occur until it was slightly modified and adopted by the National Cholesterol Education Program/ Adult Treatment Program (NCEP/ATP) for lipid lowering therapy recommendations. ${ }^{4}$ The modified Framingham Risk Score (FRS) generally categorized individuals into three CHD risk strata based on 10 year CHD risk: low risk $(<10 \%$ 10 year CHD risk), intermediate risk (10\%-20\% 10 year CHD risk) and high risk ( $>20 \% 10$ year CHD risk). The performance of the FRS in race/ethnic groups other than Caucasian and in those classified as intermediate CHD risk was suboptimal ${ }^{5-7}$ and led to significant misclassification of baseline CVD risk. This and others fueled intense research into novel risk markers and even the development of other risk scores. The overall goal of this intense research was to either identify novel risk markers which improve CHD risk prediction over and beyond the FRS or develop new risk prediction scores/tools which outperform the FRS. Thus the Reynolds risk score, SCORE, QRISK score, UKPDS score to name a few, came along. ${ }^{8-11}$ But none of the newer CVD risk scores proved to be very superior to the modified FRS and hence could not replace the modified FRS as a well-accepted alternative CHD risk prediction tool. Most of the newer risk scores also had similar discriminative ability as the FRS for CHD events despite being derived in more heterogeneous populations.

While the focus of the newer risk score developers was to replace the FRS, the focus of the novel risk marker investigators was to improve risk prediction afforded by the FRS in at least the intermediate risk group. The proliferation of population based cohort studies led to the identification of numerous novel risk markers all claiming to predict CHD risk over and beyond that afforded by the FRS. A significant number of these studies demonstrated an independent association of the novel risk marker with CHD events but did not show a significant improvement in discrimination or calibration. Eventually a top tier group of novel risk markers including coronary artery calcium score, carotid intima media thickness, ankle brachial index, high sensitivity C-reactive protein, brachial flow mediated dilation, and family history of CHD emerged. Some of these top tier novel risk markers even made it into guidelines for refining CHD risk especially in the intermediate FRS category. ${ }^{12}$ However in a head to head comparison, coronary artery calcium score appeared to be superior for refining CHD risk especially in those classified as intermediate risk by the FRS. ${ }^{13,14}$

In November 2013, the FRS, all the newer CVD risk scores that were developed in an attempt to replace the FRS, all the data on the improvement in CHD/CVD risk prediction by novel risk markers become irrelevant. The American College of Cardiology (ACC) and American Heart Association (AHA) released a new cardiovascular risk prediction tool, the new pooled atherosclerotic cardiovascular disease (ASCVD) risk estimator for calculating CVD risk in primary CVD prevention. ${ }^{15}$ Although the risk factors in the new pooled ASCVD risk estimator are similar to that in the FRS, the new pooled ASCVD risk estimator also predicts fatal and non-fatal strokes in addition to CHD. The new pooled ASCVD risk estimator was also derived and validated in a bi-racial cohort and only applicable to adults 40-79 years old unlike the FRS. The new ACC/AHA cholesterol guideline ${ }^{16}$ is heavily weighted on the new pooled ASCVD risk estimator for determining statin eligibility. Since the release of the new pooled ASCVD risk estimator, there has been a great deal of press report about it overestimating 10 year risk. ${ }^{17-19}$ There are currently a lot of ongoing analyses evaluating the improvement in discrimination that would be gained by the addition of novel risk markers to the new pooled ASCVD risk estimator in population based cohorts. Like the FRS, the new ASCVD risk estimator will remain a research target for both novel CVD risk factor investigators and new risk score developers until enough data are gathered on its performance in the real world and the degree by which these novel risk markers alter its discriminative ability is unquestionably ascertained.

In 2014, primary CVD risk prediction can be summarized as "identifying appropriate individuals for statin $+/-$ aspirin therapy" to maximize the risk-benefit ratio for adverse events such as diabetes mellitus versus preventing ASCVD events. This is so because we will always advise healthy lifestyle, blood pressure control, diabetes treatment, and cigarette smoking cessation irrespective of an individual's 10 year ASCVD risk. However, statin and aspirin have significant side effects and so we need to consider risk-benefit ratio carefully in our primary ASCVD prevention decision making. We had no idea what the risk-benefit ratio of the FRS 
and the NCEP/ATP cholesterol recommendation was with respect to statin and aspirin therapy but assumed the benefit outweighed the risk. We are yet to observe the performance of the new pooled ASCVD risk estimator and the new ACC/ AHA cholesterol guidelines.

\section{Conclusion}

If the new ASCVD risk estimator truly over estimates risk, then the lower cutoff ( $\geq 7.5 \% 10$ year ASCVD risk) for statin eligibility will mean a significant increase in the number of individuals who will be recommended statin therapy. But do we know or should we assume a net benefit as we did in the past with the FRS and the NCEP/ATP II guidelines? The jury is still out. Prospective studies addressing these concerns are needed and should be a focus for the National Institute of Health.

\section{Disclosure}

The author has no conflicts of interest to disclose.

\section{References}

1. Go AS, Mozaffarian D, Roger VL, et al. Executive summary: heart disease and stroke statistics - 2014 update: a report from the American Heart Association. Circulation. 2014;129(3):399-410.

2. Ray KK, Kastelein JJ, Boekholdt SM, et al. The ACC/AHA 2013 guideline on the treatment of blood cholesterol to reduce atherosclerotic cardiovascular disease risk in adults: the good the bad and the uncertain: a comparison with ESC/EAS guidelines for the management of dyslipidaemias 2011. Eur Heart J. 2014;35(15):960-968.

3. Kannel WB, McGee D, Gordon T. A general cardiovascular risk profile: the Framingham Study. Am J Cardiol. 1976;38(1):46-51.

4. National Cholesterol Education Program Expert Panel on Detection, Evaluation, and Treatment of High Blood Cholesterol in Adults. Executive summary of the third report of the National Cholesterol Education Program (NCEP) Expert Panel on Detection, Evaluation, and Treatment of High Blood Cholesterol in Adults (Adult Treatment Panel III). JAMA. 2001;285(19):2486-2497.

5. Brindle PM, McConnachie AM, Upton MN, Hart CL, Smith GD, Watt GCM. The accuracy of the Framingham risk score in different socioeconomic groups: a prospective study. Br J Gen Pract. 2005; 55(520):838-845.

6. Liu J, Hong Y, D'Agostino RB, et al. Predictive value for the Chinese population of the Framingham CHD risk assessment tool compared with the Chinese Multi-Provincial Cohort Study. JAMA. 2004;291(21):2591-2599.
7. D’Agostino RB Sr, Grundy S, Sullivan LM, Wilson P, CHD Risk Prediction Group. Validation of the Framingham coronary heart disease prediction scores: results of a multiple ethnic groups investigation. JAMA. 2001;286(2):180-187.

8. Ridker PM, Paynter NP, Rifai N, Gaziano JM, Cook NR. C-reactive protein and parental history improve global cardiovascular risk prediction: the Reynolds Risk Score for men. Circulation. 2008;118(22): 2243-2251.

9. Ridker PM, Buring JE, Rifai N, Cook NR. Development and validation of improved algorithms for the assessment of global cardiovascular risk in women: the Reynolds Risk Score. JAMA. 2007;297(6): 611-619.

10. Hippisley-Cox J, Coupland C, Vinogradova Y, Robson J, May M, Brindle P. Derivation and validation of QRISK, a new cardiovascular disease risk score for the United Kingdom: Prospective open cohort study. BMJ. 2007;335(7611):136.

11. Stevens RJ, Kothari V, Adler AI, Stratton IM. The UKPDS Risk Engine: a model for the risk of coronary heart disease in type 2 diabetes: United Kingdom Prospective Diabetes Study (UKPDS) Group. Clin Sci (Lond). 2001;101(6):671-679.

12. Greenland P, Alpert JS, Beller GA, et al. 2010 ACCF/AHA guideline for assessment of cardiovascular risk in asymptomatic adults a report of the American College of Cardiology Foundation/ American Heart Association Task Force on Practice Guidelines. Circulation. 2010;122(25):e584-e636.

13. Yeboah J, McClleland RL, Polonsky TS, et al. Comparison of novel risk markers for improvement in cardiovascular risk assessment in intermediate risk individuals. JAMA. 2012;308(8):788-795.

14. Kavousi M, Elias-Smale S, Rutten JH, et al. Evaluation of newer risk markers for coronary heart disease risk classification: a cohort study. Ann Intern Med. 2012;156(6):438-444.

15. Goff DC Jr, Lloyd-Jones DM, Bennett G, et al. 2013 ACC/AHA Guideline on the Assessment of Cardiovascular Risk: A Report of the American College of Cardiology/American Heart Association Task Force on Practice Guidelines. Circulation. 2014;129(25 Suppl 2): S49-S73.

16. Stone NJ, Robinson J, Lichtenstein AH, et al. 2013 ACC/AHA Guideline on the Treatment of Blood Cholesterol to Reduce Atherosclerotic Cardiovascular Risk in Adults: A Report of the American College of Cardiology/American Heart Association Task Force on Practice Guidelines. Circulation. 2014;129(25 Suppl 2):S1-S45.

17. Lenzer J. Majority of panelists on controversial new cholesterol guideline have current or recent ties to drug manufacturers. $B M J$ 2013;347:f6989.

18. Ridker PM, Cook NR. Statins: new American guidelines for prevention of cardiovascular disease. Lancet. 2013;382(9907):1762-1765.

19. Muntner P, Safford MM, Cushman M, Howard G. Comment on the Reports of Over-estimation of ASCVD Risk Using the 2013 ACC/AHA Risk Equation. Circulation. 2014;129(2):266-267.

\section{Publish your work in this journal}

Research Reports in Clinical Cardiology is an international, peerreviewed, open access journal publishing original research, reports, editorials, reviews and commentaries on all areas of cardiology in the clinic and laboratory. The manuscript management system is completely online and includes a very quick and fair peer-review system.

\section{Dovepress}

Visit http://www.dovepress.com/testimonials.php to read real quotes from published authors. 